\title{
Research of Nanosized Inhomogeneities in H-Bonded Liquids Using Sophisticated Light Scattering Techniques
}

\author{
Nataliia Kuzkova and Andrey Yakunov \\ Optics Division, Department of Physics, Taras Shevchenko National University of Kyiv, 4 Glushkova Avenue, Building 1, \\ Kyiv 03127, Ukraine
}

Correspondence should be addressed to Nataliia Kuzkova; natali_lizz@ukr.net

Received 15 June 2013; Accepted 24 July 2013

Academic Editors: V. Kochereshko and S. Krukowski

Copyright (c) 2013 N. Kuzkova and A. Yakunov. This is an open access article distributed under the Creative Commons Attribution License, which permits unrestricted use, distribution, and reproduction in any medium, provided the original work is properly cited.

\begin{abstract}
Hydrogen bonded (H-bonded) liquids can be considered as mediums that have nanosized heterogeneities. Previously, it was shown that the light scattering by acoustic phonons is accompanied by fluctuations of intensity of light with power spectral density $S(f) \sim$ $1 / f^{\alpha}(\alpha \approx 1)$ that reflects the phonon energy fluctuations. In this work fluctuations of light scattering in H-bonded liquids have been investigated. We consider two mechanisms of $1 / f$-process forming: fluctuations of phonon energy and fluctuations caused by dynamical inhomogeneities, predicted by percolation model. The $\alpha$ variability can be explained by different contributions of both scattering mechanisms, which form an overall picture of low-frequency fluctuations of scattering light intensity in complex liquids.
\end{abstract}

\section{Introduction}

Slow fluctuations of intensity of scattered light in liquids, observed in several experiments [1-3], indicate the existence of a long-range correlation of molecular vibrations, which creates conditions for the slow evolution and stability of local nanosized structural formations. Power spectral density of these fluctuations is described by the law $S(f) \sim 1 / f^{\alpha}$, where $\alpha$ is close to 1 . It gives grounds to attribute them to a wide class of phenomena known as " $1 / f$-fluctuations," which is distributed in nature and represents an interest for research. It is considered that $1 / f$ fluctuations in condensed matter are caused by the existence of specific structural and functional anomalies (nonlinearity, metastable states, faults, traps, and clusters) that have a wide range of lifetimes [4].

A cycle of works [5-8] had been performed by Musha and Borbely. An original two-beam optical scheme was used. It was shown that the light scattering by acoustic phonons is accompanied by $1 / f$ fluctuations of intensity and the law reflects the phonon energy fluctuations. Light scattering in the liquid systems (water, electrolyte solutions) occurs on phonons localized at inhomogeneities (clusters). It can be expected that fluctuations of intensity of light represent characteristics of the centers of localization and, therefore, give additional information on nanosized structure of liquid systems.

Based on the modified Musha-Borbely scheme, we investigate intensity fluctuations of scattered light in hydrogenbonded liquids: water, glycerol, and their binary solutions. Water-glycerol solutions are known as heterogeneous systems with complex hierarchical structure and multiple anomalies [9-11].

\section{Methodology and Experimental Measurements}

As the light waves pass through condensed matter, the secondary radiation can be observed. It is caused by scattering and luminescence. Overall picture of light scattering includes (a) high-frequency inelastic (Raman) scattering on optical phonons, (b) low-frequency inelastic (BrillouinMandelstam) scattering on acoustic phonons, and (c) elastic (Rayleigh) scattering that occurs in structural inhomogeneities (i.e., clusters). 
It is known [12] that Raman scattering and luminescence are isotropic for liquids. Rayleigh scattering on centers which are much smaller than the wavelength is symmetrical about the normal to the propagation of the beam. In the general case Rayleigh and Brillouin-Mandelstam scatterings have asymmetric angular distribution [13, 14]. The greater the size of scattering centers, the stronger the dissymmetry.

Selection of the contribution of scattering on largesize inhomogeneities among other types of secondary radiation is possible by measuring the difference in intensities of the two secondary waves that are distributed at angles $90 \pm \varphi$ relatively, to the direction of the primary wave.

This idea has been implemented by using an experimental setup which is shown in Figure 1. Multimode semiconductor laser at the wavelength $\lambda=409 \mathrm{~nm}$ (output power $60 \mathrm{~mW}$ ) was used as a light source. The spectral width of the main mode was $\Delta \lambda \approx 0.04 \mathrm{~nm}$. Adjacent modes were less intensive, so they were neglected.

Focused laser beam was injected into the cuvette with the liquid. We used disposable poly cuvettes $10 \times 20 \times 50 \mathrm{~mm}$ and wall thickness $0.5 \mathrm{~mm}$. The amount of the distance from the edge of slit to laser beam in the specimen was $1 \mathrm{~mm}$. After passing through the sample, the laser beam was absorbed by trap in order to prevent the return of the reflected light. The scattered light from the sample passed through the slit and felled on the screen with two round holes (double aperture) located at a distance $2 \mathrm{~cm}$ on both sides of the optical axis. The distance from slit to screen was $11 \mathrm{~cm}$. Thus, scattering was registered at the angles $90^{\circ} \pm 10^{\circ}$.

The beams of light passed through the lens $(F=$ $6 \mathrm{~cm}$ ). Then a special modulator (modulation frequency $200 \mathrm{~Hz}$ ) directed them on a photomultiplier (PMT) one by one. The violet filter was set before the PMT in order to remove the light caused by Raman scattering and luminescence. The difference of intensities was registered as a variable component of the signal with a frequency $200 \mathrm{~Hz}$.

A special disk compensator for additional balance of channels in optical scheme was implemented. A cuvette with a water solution of dye for configuration and calibration of the setup was used. The luminescence signal was registered. It is characterized by the isotropic angular distribution of light intensity. The concentration of dye led to luminescence intensity $I_{0}^{L}$, which is approximately two orders higher than the intensity of scattering $\left(I_{0}^{S}\right)$ on $80 \%$ water glycerol solution.

The amount of isotropic angular distribution $\left(\Delta I^{L} / I_{0}^{L}\right)$ of luminescence signal was $10^{-4}$ a.u. For isotropic scattering, differential signal $\left(\Delta I^{S}\right)$ was below the noise level. In our experiments, all signals reliably exceeded this level, which evidenced the dissymmetry of angular distribution of scattering intensity for the investigated samples.

Further signal processing was performed by using selective amplifier, analog-digital converter, and personal computer. Total number of counts per registration procedure

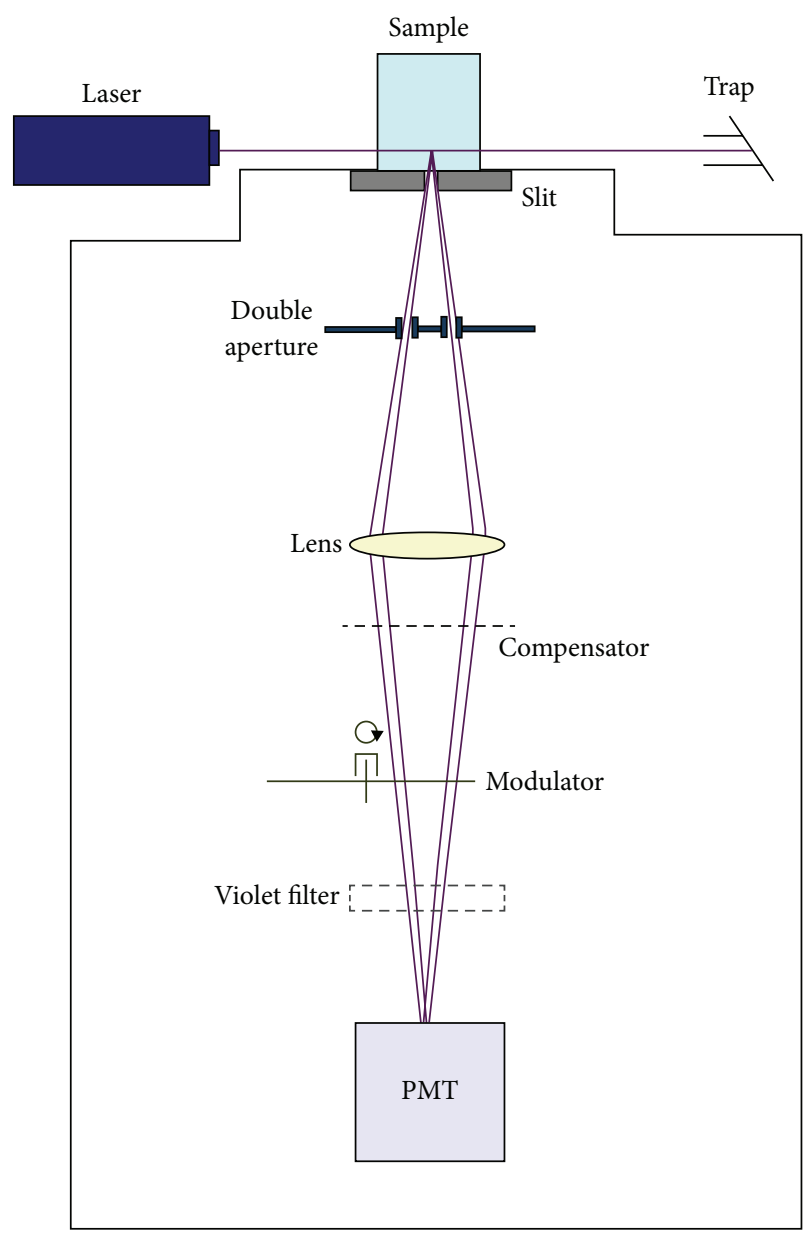

FIGURE 1: Experimental setup.

was $4 \cdot 10^{5}$. Using Fourier transform for each time series a power spectrum was obtained:

$$
S(f) \sim\left|\int_{0}^{T} U(t) \cdot e^{i \cdot 2 \pi f t} d t\right|^{2}
$$

Power spectrum in low-frequency range was approximated by the function $S(f)=C / f^{\alpha}$.

We used standard distilled water for medical purpose and chemically pure glycerol. Water solutions of glycerol with volumetric concentrations were prepared: $1 \%, 2 \%, 5 \%, 10 \%$, $20 \%, 40 \%$, and $80 \%$. The accuracy of dilution was $1 \%$. Before the measurements, specimens were sustained at least one day to avoid the influence of the slow dynamics of structure formation [15].

\section{Results}

Figure 2 shows the power spectral density of intensity fluctuations of scattered light in distilled water and pure glycerol. The figure illustrates that both spectra in the frequency range $0.1-50 \mathrm{~Hz}$ are described by the function $S(f) \sim 1 / f^{\alpha}$. The $\alpha$ value for water $(\alpha=1,12)$ coincides 


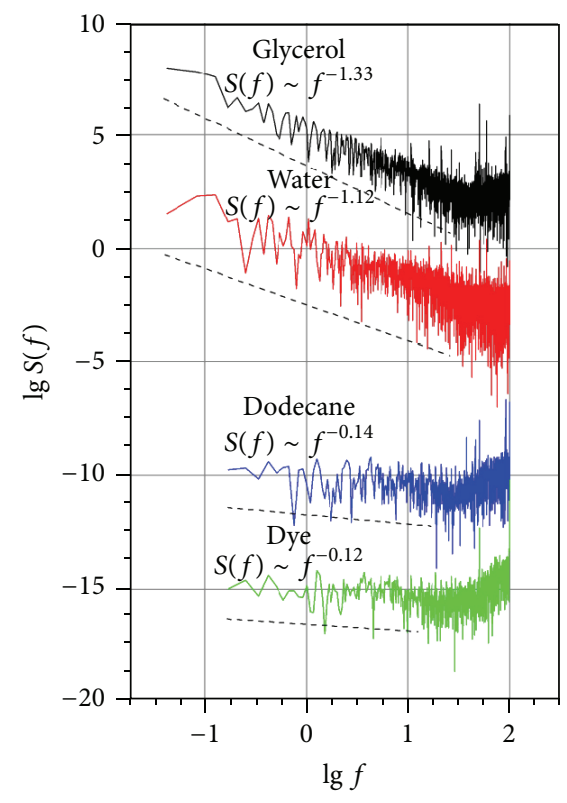

FIgURE 2: Power spectral density of intensity fluctuations of scattered light in distilled water, glycerol, dodecane, and fluorescence of dye.

with the data [6]; at the same time we get the $\alpha$ value in glycerol $(\alpha=1,33)$ greater than liquids listed in the work [8].

For comparison, in the same way, we measured intensity fluctuations of scattered light for liquid without hydrogen bonds-dodecane $\left(\mathrm{C}_{12} \mathrm{H}_{26}\right)$. It turned out that power spectral density does not differ from the corresponding fluorescence spectrum. This confirms data [8] that not for all liquids intensity fluctuations of scattered light is observed.

Figure 3 presents the $\alpha$ value dependence of concentration for the water-glycerol solution. It corresponds with well-known water-glycerol solution anomalies [9] and agreements with the data of dielectrometry [10] and spectroscopy [11].

It is known that water-glycerol solution has three structural phases, "depleted," transient, and "enriched" ones, which is characteristic of micellar systems. In other words, the structure of solution at low and high concentrations of one of the components is realized in the form of coexistent solvent matrix and nanosized associates of other liquids. Our results prove previous data [11] about the existence of a critical concentration, where the structure of water-glycerol solution is realized in the form of matrix and nanodimensional associates.

The sharp increase in the parameter $\alpha(C>15 \%)$ we connect with the inroduction of another scattering mechanism associated with the appearance of nanosised scattering centers. The structural parameter $\alpha$ can be very efficient and intuitive, and provide the new information in the comparison of low-frequency vibrational spectra of liquids with different

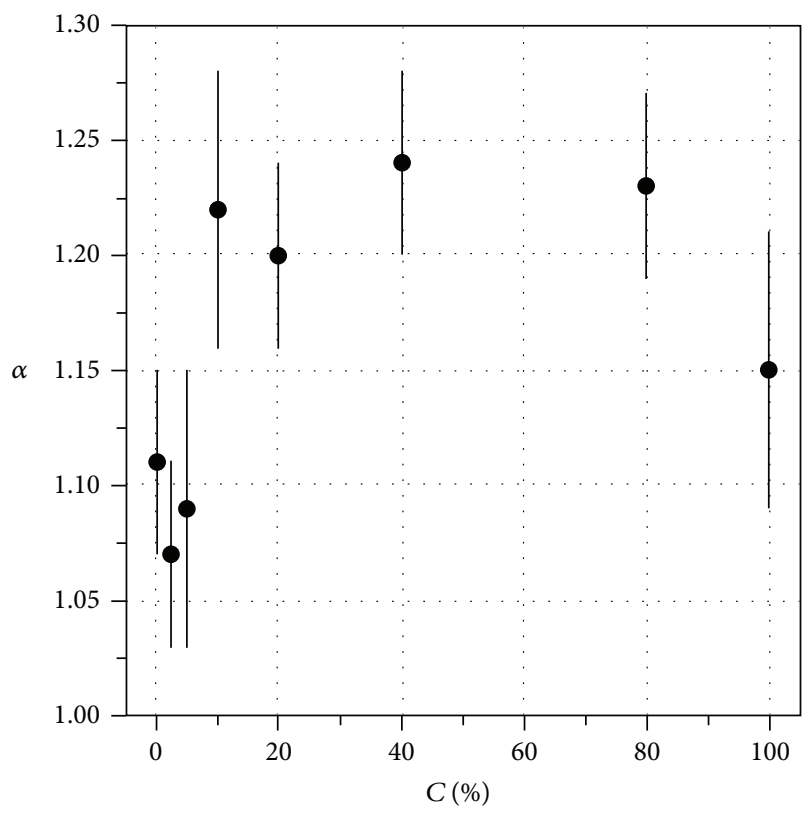

Figure 3: Concentration dependence of index $\alpha$ for glycerol in water-glycerol solution.

molecular organization, but with the same type of connection $[3]$.

\section{Models}

We consider two mechanisms of $1 / f$-process forming: fluctuations of phonon energy and fluctuations caused by dynamical inhomogeneities, predicted by percolation model. Thus, they represent the Brillouin-Mandelstam scattering and the Rayleigh one correspondingly.

It is known that $1 / f$-process is observed in nonlinear systems in which intermittency chaos can be realized. In this respect, the one-dimensional vibrating systems of particles coupled by nonlinear forces, proposed by Fermi, Pasta, and Ulam (FPU) are a good test ground [16]. The FPU model is simple and general enough to describe the fundamental properties that nonlinear lattices have. Behavior of nonlinear chain was studied by Easy Java Simulation software.

The FPU equation is composed of harmonic linear and small nonlinear terms.

Taking into account only nearest interactions we obtain the following equation:

$$
\begin{aligned}
m \frac{\partial^{2} y_{i}}{\partial t^{2}}= & c\left(y_{i+1}-2 y_{i}+y_{i-1}\right) \\
& +k\left[\left(y_{i+1}-y_{i}\right)^{3}-\left(y_{i}-y_{i-1}\right)^{3}\right],
\end{aligned}
$$

where $i$ runs from 1 to $N-1$ with $y_{0}=y_{N}=0$ for $N+1$ particles with equal masses $m$ of a chain with fixed ends, $c$ and $k$-linear and nonlinear elastic constants. 
The phonon energy belonging to a certain mode $q$ of vibration is proportional to the portion $E_{q}(t)$ of the total vibration energy and can be approximated in the previous case by

$$
E_{q}(t)=\frac{1}{2} \dot{a}_{q}^{2}(t)+2 a_{q}^{2}(t) \cdot \sin ^{2} \frac{\pi q}{2 N},
$$

where $a_{q}(t)$ is the spatial Fourier coefficient of the particles displacements at the time $t$.

In linear chain $E_{q}(t)$ is identified with the number of excited phonons. We have investigated $E_{q}(t)$ at a fixed $k$ to study the phonon number of fluctuations. $E_{q}(t)$ oscillates irregularly with $1 / f$ dependence even for small values of $k$ (Figure 4). The parameters of spectrum depend weakly on $k$. For the systems with phonon mechanism of fluctuations, the $\alpha$ index has approximately the same value, which agrees with works [5-8].

Intensity of the light scattering at a fixed angle is proportional to the density of vibrational states at the appropriate frequency. Hence the fluctuations of the energy of some mode cause fluctuations of intensity of scattered light in a given mode.

It can be said that the fluctuations have not thermodynamic, but nonlinear dynamic nature which reflect the chaotic behavior of a nonlinear system. A slow relaxation process due to the anharmonic connection between the phonons gives rise to long-term fluctuations around thermal equilibrium.

The second model is considering the Rayleigh light scattering on nanosized structures. Such structures are nanosized domains with a peculiar, different from the surrounding structure packing of molecules. According to percolation model [17], structure of H-bonded liquid can be presented as an infinite cluster, where cavities are occupied by smaller clusters and individual molecules (Figure 5). Distribution of cavity size depends on temperature and impurities. The average sizes of inhomogeneities are determined by the length correlation.

It should be noted that the existence of nano- and micro scattering centers in the pure liquids is predicted in other models, for instance, in a cluster model [14]. Sizes of the clusters obtained from the calculations have the order of correlation length in percolation model [11].

The dynamic nature of the structure points on the fact that in each moment of time the distribution of cavity size is unique. Possible approach to the modeling of this phenomenon can be explained by cellular automata [18]. Modeling was performed using the original "Cellular Matter" program [19]. Each iteration of cellular model leads to some realization of the instantaneous structure of the infinite cluster. It was found that the distribution obeys the exponential law (Figure 6).

In each of the next iterations some changes occurred. Spatial and temporal scale invariance which is characteristic for percolation structures results in the fact that light scattered on such structures is followed by fluctuations, the spectrum of which is described by the same exponential law.

Nanosized inhomogeneities make the second-order correction in the overall picture of the elastic light scattering

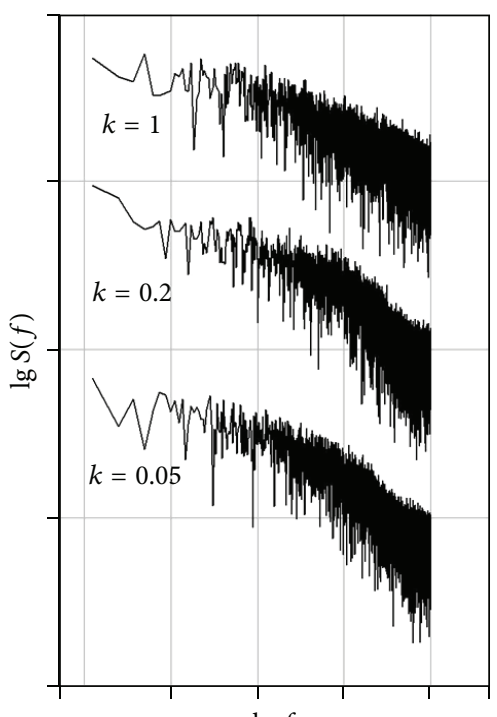

$\lg f$

Figure 4: Power spectral density of phonon energy fluctuations in a chain of nonlinear coupled particles.

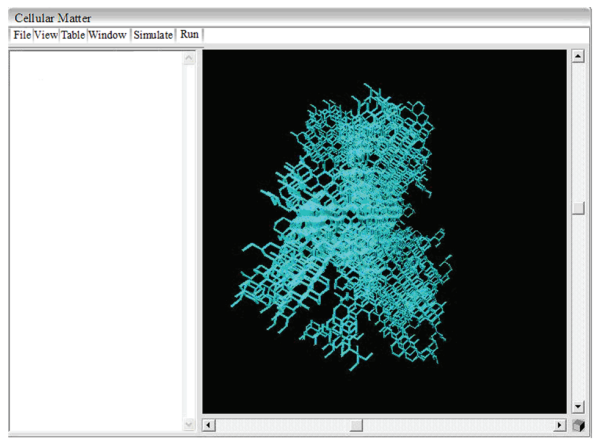

Figure 5: Percolation cluster on the three-dimensional tetrahedral lattice [11].

[15]. In a case, when inhomogeneities commensurate with the wavelength, this correction has a considerable impact on angular distribution [14].

\section{Discussion}

According to the statements described above, the fluctuations are caused by both of the processes. The weight is determined by the coefficient defined by the Landau-Placzek relation of unshifted (1-Rayleigh) and shifted (2-Brillouin-Mandelstam) components of the scattering [12]:

$$
\frac{I_{1}}{2 I_{2}}=\frac{\beta_{T}-\beta_{S}}{\beta_{S}},
$$

where $\beta_{T}$ is the isothermal compressibility and $\beta_{S}$ is the adiabatic compressibility. $I_{1}$ is determined by the isobaric density fluctuations and $I_{2}$ by adiabatic density fluctuations.

It is possible to predict in which type of liquid a certain type of scattering will be dominant. For pure homogeneous 


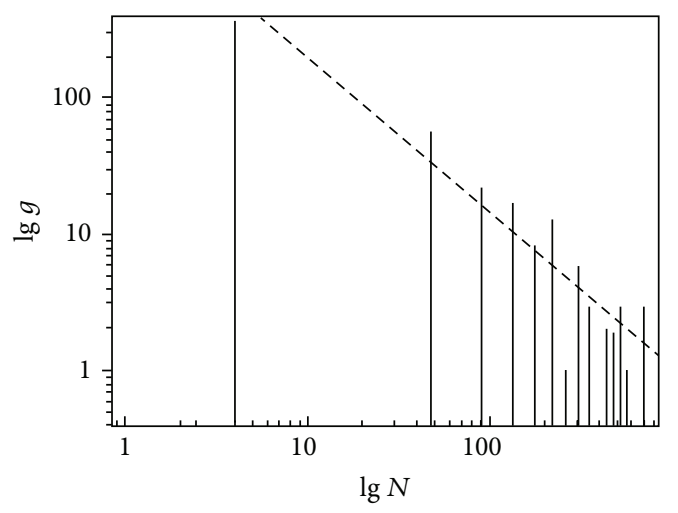

(a)

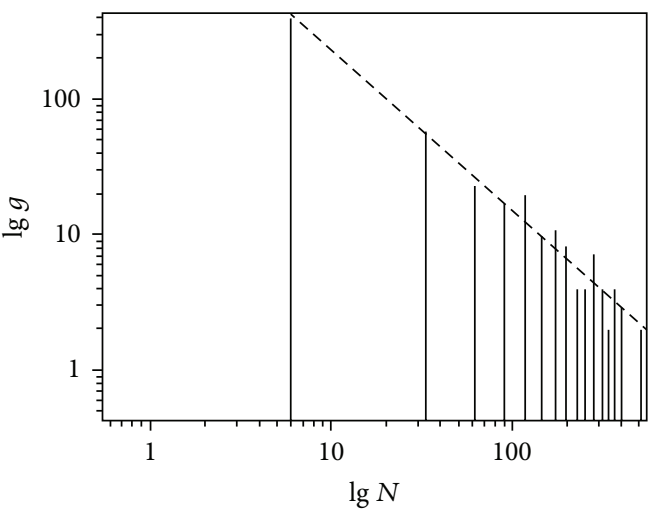

(b)

FIgURE 6: Distribution of cavities according to their size.

liquids, process (2) is dominating [8], and the contribution of process (1) can be neglected, so $\alpha \approx$ const for liquids which corresponds to the model mentioned above of the nonlinear lattice. For inhomogeneous $\mathrm{H}$-bonded liquids processes (1) and (2) provide commensurate contribution, and $\alpha$ may depend on the type of liquid in accordance with the distribution law of inhomogeneities in percolation cluster.

Dodecane is a homogeneous liquid without hydrogen bonds for which $I_{1} / 2 I_{2}<1$ [20]. There dominates Rayleigh scattering by isobaric density fluctuations only. Thence fluctuations of light scattering in pure Dodecane (also in other liquids with similar properties [8]) do not manifest and prove our results.

At the same time, for pure water at room temperature $I_{1} / 2 I_{2}>0$; this makes the contribution to BrillouinMandelstam scattering fluctuations dominant. Therefore, index $\alpha$ is close to 1 , as in the works [6,8]. For other liquids (i.e., glycerol) with $\mathrm{H}$-bonds, with value of $I_{1} / 2 I_{2} \sim 1$, the fluctuations affect both processes equally.

Our results indicate the good perspectives for $1 / f$ spectroscopy method. Although such spectra are structureless, their approximation by $S(f)=C / f^{\alpha}$ gives two independent parameters $C$ and $\alpha$. They have information about fluctuations of the scattering cross section and fluctuations of the number of scatterers centers in the bulk, that is, about dynamic characteristics of nanosized structure.

\section{Conclusions}

A set of low-frequency fluctuations of the intensity of light scattered by the liquid hydrogen-bonded systems (distilled water, glycerol, and water-glycerol solutions) was measured. The calculation of the power spectral density corresponds to time series in the range of $0.1-50 \mathrm{~Hz}$ for the statement $S(f) \sim 1 / f^{\alpha}$, where $\alpha \approx 1$.

We consider two mechanisms of $1 / f$-process forming: fluctuations of phonon energy and fluctuations caused by dynamical inhomogeneities. Thus, they represent the Brillouin-Mandelstam scattering and the Rayleigh one correspondingly.
We found that index $\alpha$ depends on the type of liquid and solution concentration. This dependence can be explained by different contributions of both scattering mechanisms, which form an overall picture of low-frequency fluctuations of scattering light intensity in complex liquids.

\section{References}

[1] F. R. Chernikov, "Influence of certain physical factors on the fluctuations of light scatter in water and aqueous solutions of biopolymers," Biophysics, vol. 35, no. 5, pp. 717-721, 1990.

[2] V. I. Lobyshev, B. D. Ryzhikov, and R. E. Shikhlinskaya, "Spontaneous and external electromagnetic field-induced long-term transient processes in diluted aqueous tryptophan solutions and water," Biophysics, vol. 43, no. 4, p. 715, 1998.

[3] L. Bulavin, A. Yakunov, and M. Bily, "Manifestation of slow fluctuations of water structure in the spectra of Raman light scattering," Physics of Alive, vol. 13, no. 1, pp. 43-50, 2005.

[4] M. B. Weissman, "1/f noise and other slow, nonexponential kinetics in condensed matter," Reviews of Modern Physics, vol. 60, no. 2, pp. 537-571, 1988.

[5] M. Toshimitsu, B. Gãbor, and S. Minoru, "1/f phonon-number fluctuations in quartz observed by laser light scattering," Physical Review Letters, vol. 64, no. 20, pp. 2394-2397, 1990.

[6] T. Musha and G. Borbely, "1/f fluctuations of phonon energy in water," Japanese Journal of Applied Physics B, vol. 31, no. 3, pp. L370-L371, 1992.

[7] T. Tohei, K. Nakagawa, K. Takada, and T. Musha, "1/f fluctuations in $\mathrm{LiCl}$ solution," Japanese Journal of Applied Physics, vol. 35, no. 3, pp. 1781-1785, 1996.

[8] G. Borbely, "1/f phonon number fluctuations in pure liquids," Journal of Communications, vol. 46, no. 2, pp. 32-35, 1995.

[9] I. I. Adamenko, L. A. Bulavin, V. Ilyin, S. A. Zelinsky, and K. O. Moroz, "Anomalous behavior of glycerol-water solutions," Journal of Molecular Liquids, vol. 127, no. 1-3, pp. 90-92, 2006.

[10] A. Puzenko, Y. Hayashi, and Y. Feldman, "Space and time scaling in glycerol-water mixtures," Journal of Non-Crystalline Solids, vol. 353, no. 47-51, pp. 4518-4522, 2007.

[11] A. O. Maksymov, A. V. Yakunov, and M. M. Bily, "Investigation of H-bonded media by means of Raman scattering in terms of the fractal formalism," in Nanophotonic Materials VI, Proceedings of SPIE, San Diego, Calif, USA, August 2009. 
[12] I. L. Fabelinskii, Scattering of Light, Nauka, Moskow, Russia, 1965.

[13] T. Still, High Frequency Acoustics in Colloid-Based Mesoand Nanostructures by Spontaneous Brillouin Light Scattering, Springer, Berlin, Germany, 2010.

[14] V. F. Kovalenko, P. G. Levchenko, S. V. Shutov, and A. Y. Bordiuk, "Investigation of the nature of light scattering by water," Ukrainian Journal of Physical Optics, vol. 10, no. 1, pp. 38-53, 2009.

[15] M. Sedlák, "Large-scale supramolecular structure in solutions of low molar mass compounds and mixtures of liquids. I. Light scattering characterization," Journal of Physical Chemistry B, vol. 110, no. 9, pp. 4329-4338, 2006.

[16] K. Fakamachi, "1/f fluctuations in FPU lattices", Europhysics Letters, vol. 26, no. 6, article 449, 1994.

[17] T. DeSimone, S. Demoulini, and R. M. Stratt, "A theory of percolation in liquids," The Journal of Chemical Physics, vol. 85, no. 1, pp. 391-400, 1986.

[18] L. B. Kier and C.-K. Cheng, "A cellular automata model of water," Journal of Chemical Information and Computer Sciences, vol. 34, no. 3, pp. 647-652, 1994.

[19] A. Yakunov and P. Yakunov, "Slow dynamics of water structure in cellular automata model," in Proceedings of the International Conference on Physics of Liquid Matter: Modern Problems (PLMP '05), p. 140, Kyiv, Ukraine, May 2005.

[20] E. Gray, American Institute of Physics Handbook, McGraw-Hill Book Company, New York, NY, USA, 1972. 

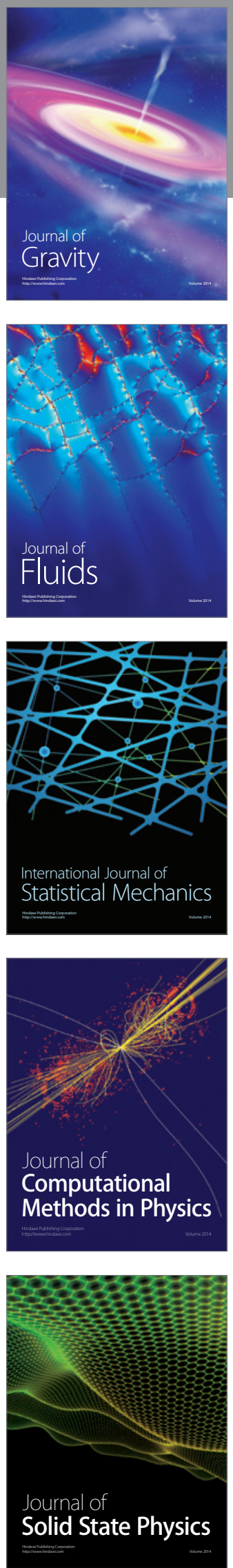

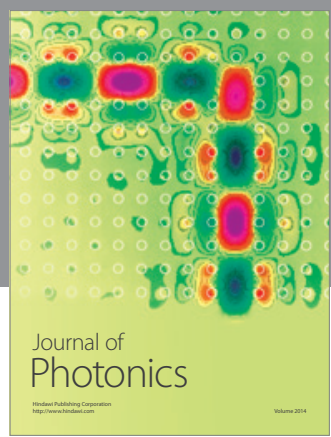

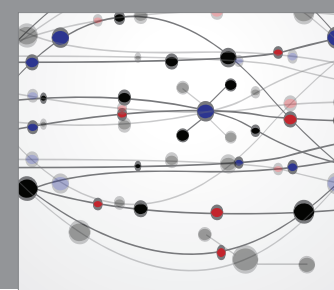

The Scientific World Journal

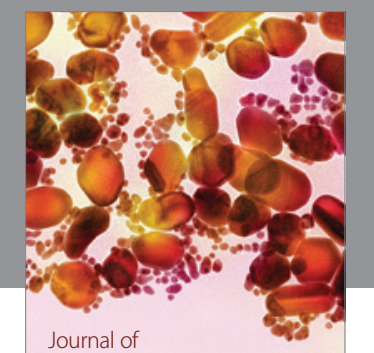

Soft Matter
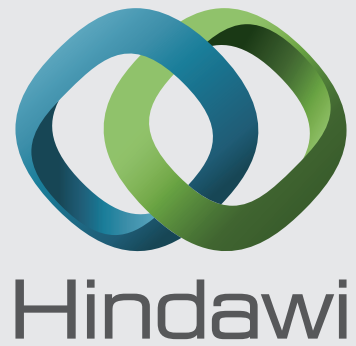

Submit your manuscripts at

http://www.hindawi.com
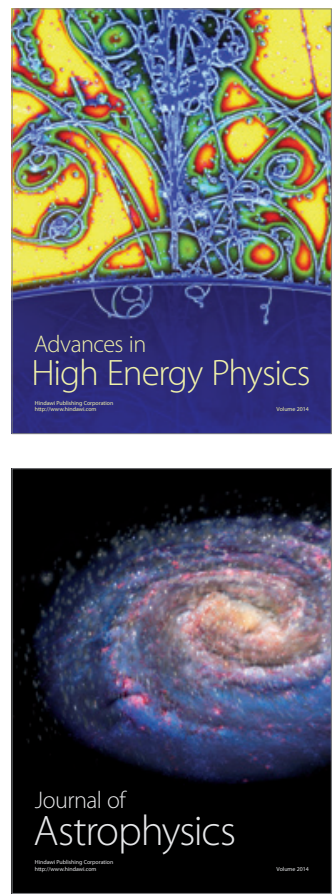
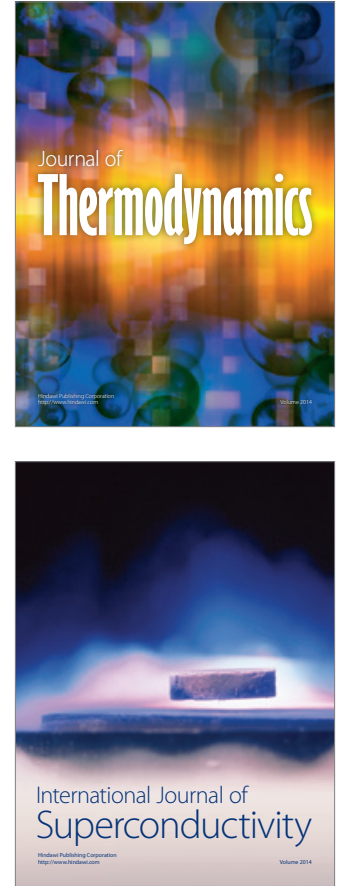
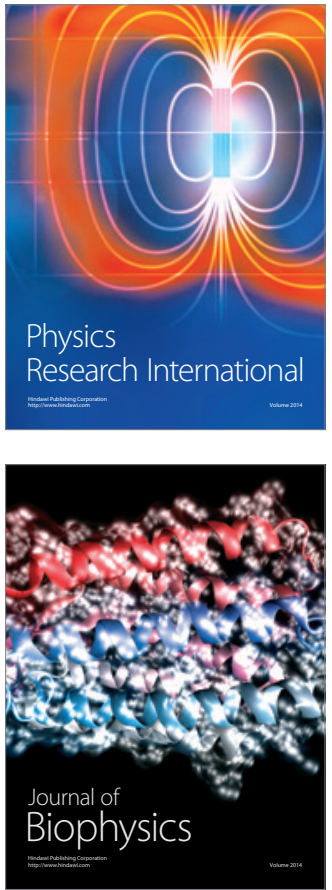
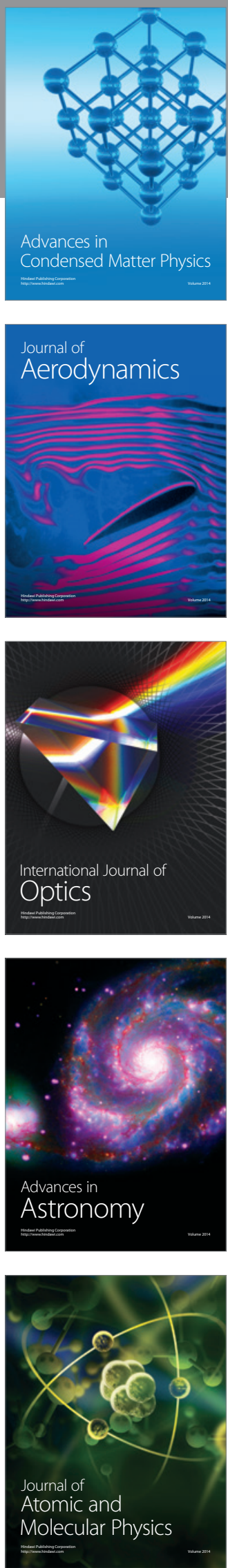